\section{Venetoclax in combination with carfilzomib, doxorubicin and dexamethasone restores responsiveness in an otherwise treatment-refractory multiple myeloma patient}

Multiple myeloma (MM) is characterized by the clonal expansion of plasma cells (PC) in the bone marrow (BM). Over the last decade, the outcome of MM patients has substantially improved due to the introduction of several novel anti-MM agents, including proteasome inhibitors (PI: bortezomib (V), carfilzomib (K), ixazomib), immunomodulatory drugs (IMiD: thalidomide, lenalidomide (R), pomalidomide) and monoclonal antibodies (elotuzumab, daratumumab [Dara]). However, long-term therapy efficacy and tolerability remain challenging in the treatment of relapsed and refractory MM (RRMM). ${ }^{1}$ Therefore, innovative strategies and combination therapies are critically needed.

A balance between anti-apoptotic (BCL-2, BCL-XL, MCL-1) and pro-apoptotic (BAX, BAK, NOXA) proteins is required to regulate the intrinsic apoptotic pathway. ${ }^{2}$ Overexpression of anti-apoptotic proteins such as BCL-2 can be found in a subset of MM cells. ${ }^{3}$ Venetoclax is a selective and potent BCL-2 inhibitor used effectively in several hematological malignancies, including chronic lymphocytic leukemia and non-Hodgkin lymphoma. ${ }^{4}$ In a phase I clinical trial (NCT01794520), venetoclax monotherapy demonstrated anti-MM activity in RRMM with translocation $\mathrm{t}(11 ; 14)$.

Here, we describe a patient with aggressive, prognostically unfavorable MM with high BM infiltration, extramedullary (EM) relapses and refractoriness to $\mathrm{V}, \mathrm{K}$, bendamustin (B), Dexamethasone (D) and Dara, with intolerance of lenalidomide. However, treatment with KDoxorubicin (D)-D plus continuous low-dose venetoclax resulted in a major response.

In $05 / 2014$, a 53-year-old man was referred to our tertiary hospital with weakness, exertional dyspnea and bone pain and a 5-year history of smoldering MM (SMM) diagnosed in an outpatient hematology/oncology practice in 09/2009. The initial BM PC infiltration had been $16 \%$. Lacking hypercalcemia, renal failure, anemia and/or bone lesions (CRAB) features at that time, he was managed with "watchful waiting", while serological SMM markers were slowly progressing.

In $05 / 2014$, sudden disease progression to ISS stage III IgG kappa ( $\mathrm{\kappa})-\mathrm{MM}$ was confirmed (Figure 1A): he presented with acute renal failure (serum creatinine, $4.11 \mathrm{mg} / \mathrm{dL})$, hypercalcemia $(3 \mathrm{mmol} / \mathrm{L})$ and anemia (hemoglobin: $8.7 \mathrm{~g} / \mathrm{dL}$ ). A low-dose whole body computerized tomography (WB-CT) scan demonstrated extensive osteolytic lesions throughout the entire skeleton. Magnetic resonance imaging (MRI) revealed thoracic spine disease with extra-osseous soft tissue invading the neural foramina T2/3 and T4/5. The IgG level was 6547 $\mathrm{mg} / \mathrm{dL}$ (normal range $[\mathrm{NR}]<1250 \mathrm{mg} / \mathrm{dL}$ ) and serum immunofixation confirmed monoclonal IgGк. Serum free light chain (SFLC) quantification (Freelite ${ }^{\mathrm{TM}}$ ) showed highly elevated $\kappa$-SFLC of $1300 \mathrm{mg} / \mathrm{L}(\mathrm{NR}:<19.4)$, and BM-cytology and -histology demonstrated dense (90\%) PC infiltration. Fluorescence in-situ hybridization (FISH) confirmed high-risk features with $\mathrm{t}(11 ; 14) / \mathrm{IGH}-\mathrm{CCND} 1$ and gain of 1q21 in $80 \%$ of analyzed interphase nuclei. Most of those cells $(75 \%)$ showed one additional IgHCCND1 fusion signal.

Induction with three cycles of cyclophosphamide (C)VD (VCD) was performed..$^{5}$ Additionally, he received radiation therapy to treat thoracic soft tissue $\mathrm{MM}$ lesions.
In 09/2014, an autologous stem cell transplantation (ASCT) was performed after conditioning with 200 $\mathrm{mg} / \mathrm{m}^{2}$ melphalan. Thereafter, substantial reduction of PC BM infiltration $(<10 \%)$ was obtained and all MM specific markers (IgG, $\kappa$-SFLC) returned to normal (very good partial response [VGPR] according to International Myeloma Working Group [IMWG] criteria). Maintenance was at that time not approved and a clinical trial offering maintenance ${ }^{6,7}$ was refused by the patient.

Regular follow-up post ASCT was performed and the patient remained in VGPR. However, 2 years after ASCT he developed cervical pain and MRI showed new extradural cervical vertebrae (CV6 pedicle) MM infiltration compressing the C6 nerve root. Serological markers had slightly increased (к-SFLC $23.8 \mathrm{mg} / \mathrm{L}$, IgG 1346 $\mathrm{mg} / \mathrm{dL}$ ), but BM-cytology and -histology continued to show $<10 \%$ PC. Due to his EM relapse, irradiation of CV6 was performed and the patient received $\mathrm{Rd}$. Unfortunately, five days after $\mathrm{Rd}$ initiation, severe CTCAE grade 3 skin toxicity, ${ }^{8}$ very likely related to $\mathrm{Rd}$, occurred and Rd was stopped. Instead, he received continuous second line therapy (LT) with VDD,,${ }^{9,10}$ followed by conditioning with melphalan $\left(200 \mathrm{mg} / \mathrm{m}^{2}\right)$ - bortezomib $\left(2 \mathrm{mg} / \mathrm{m}^{2}\right)^{11}$ and second ASCT in 09/2016. Thereafter, maintenance therapy was applied with VD. ${ }^{6,7}$

Three years after the second ASCT, the patient presented with increased serological markers ( $\kappa$-SFLCs 25 $\mathrm{mg} / \mathrm{L}), \mathrm{BM}$ infiltration of $80 \%$, and $\mathrm{t}(11 ; 14)$ and gain 1q21 confirmed by FISH. Eight-color multiparameter flow cytometry (MFC; Figure 1B) revealed aberrant PC (aPC) $(16 \%$ of cells). The aPC population showed a $\mathrm{CD} 138^{+} \mathrm{CD} 38^{\mathrm{dim}+} \mathrm{CD} 19^{\mathrm{dim}-} \mathrm{CD} 45^{\mathrm{dim}-} \mathrm{CD} 56^{-} \mathrm{CD} 27^{-}$phenotype and monoclonal $\mathrm{K}$ expression.

Due to this second progression, the third LT with DaraVD was started. ${ }^{12}$ This combination therapy, however, failed to achieve any MM response, and due to rapid progression the fourth LT with KBD was performed according to the EMN-09 trial. ${ }^{13}$ Unfortunately, further MM progression occurred (increased $\kappa-S F L C$ and $\operatorname{IgG}$ ) and new extensive bone and EM MM lesions were detected via WB-CT and MRI. Due to his initial response to VDD, the fifth LT with KDD was started and due to the presence of $\mathrm{t}(11 ; 14)$ on FISH analysis, treatment was augmented with low-dose venetoclax (Ven) (100 mg/day). This quadruplet therapy was well tolerated and serological VGPR was achieved (к-SFLC $34.5 \mathrm{mg} / \mathrm{L}$, IgG 1058 $\mathrm{mg} / \mathrm{mL}$ )

At present (07/2019), the patient continues to receive KDD-Ven, no drug-related adverse events (AE) have occurred, the patient's bone pain has completely resolved and his quality of life (QoL) substantially increased (revised myeloma comorbidity index [R-MCI] improved from $6 / 9=$ intermediate-fit $\rightarrow$ to $3 / 9=$ fit). ${ }^{14,15}$

The BM-re-biopsy confirmed excellent response to KDD-Ven, with no residual PC expansion. The MRD flow cytometry analysis via 8-color-MFC (Figure 1C) revealed residual PC in $0.004 \%$ (of 900,000 cells). The

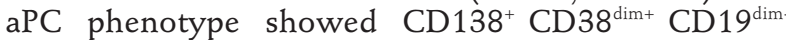
CD45 ${ }^{\text {dim- }}$ CD56-CD27- expression and $\mathrm{K}$-monoclonality. The patient was classified as MRD- with $10^{-4}$, but not reaching $10^{-5}$, due to a hypocellular $\mathrm{BM}$ aspirate, as it had not been possible to acquire 3,000,000 cells for MFC.

In the phase I clinical trial (NCT01794520) venetoclax monotherapy demonstrated anti-MM activity in RRMM with a translocation $\mathrm{t}(11 ; 14) .{ }^{4}$ As shown in vitro, Dexamethasone promotes BCL-2- dependence in MM resulting in sensitivity to venetoclax. ${ }^{4}$ In vitro PI can upregulate the MCL-1 inhibitor NOXA leading to functional neutralization of MCL- $1 .{ }^{4}$ In a phase $1 \mathrm{~b}$ trial (NCT 
01794507) with venetoclax and bortezomib in RRMM, patients refractory to bortezomib were less likely to respond compared to those who were exposed but not refractory to bortezomib. ${ }^{16}$ Since the next generation PI carfilzomib is more potent than bortezomib ${ }^{4,17}$ and our patient had acquired bortezomib-resistance, quadruplet therapy of KDD-Ven was started.

In the NCT017945078 trial combining Ven-VD, venetoclax doses ranged from $100 \mathrm{mg}$ to $1200 \mathrm{mg} /$ day ${ }^{16}$ In a recent combination therapy trial (M15-654) with DaraVD-Ven, venetoclax doses were 400 or $800 \mathrm{mg}$. An association between higher venetoclax exposures and higher neutropenia rates in $\mathrm{MM}$ patients was confirmed. ${ }^{16}$ Due to pancytopenia after the fourth LT in our patient, we decided to continue venetoclax at a low dose (100 $\mathrm{mg} /$ day). This may have also been advantageous in the light of the phase III BELLINI trial (M14-031) that was transiently stopped due to the higher proportion of deaths in the venetoclax compared to the control arm, which was mainly caused by infections. The Food and Drug Administration had therefore placed a partial hold on all clinical trials evaluating venetoclax for the investigational treatment of MM. Despite these results, numerous venetoclax monotherapies and more recently combi-
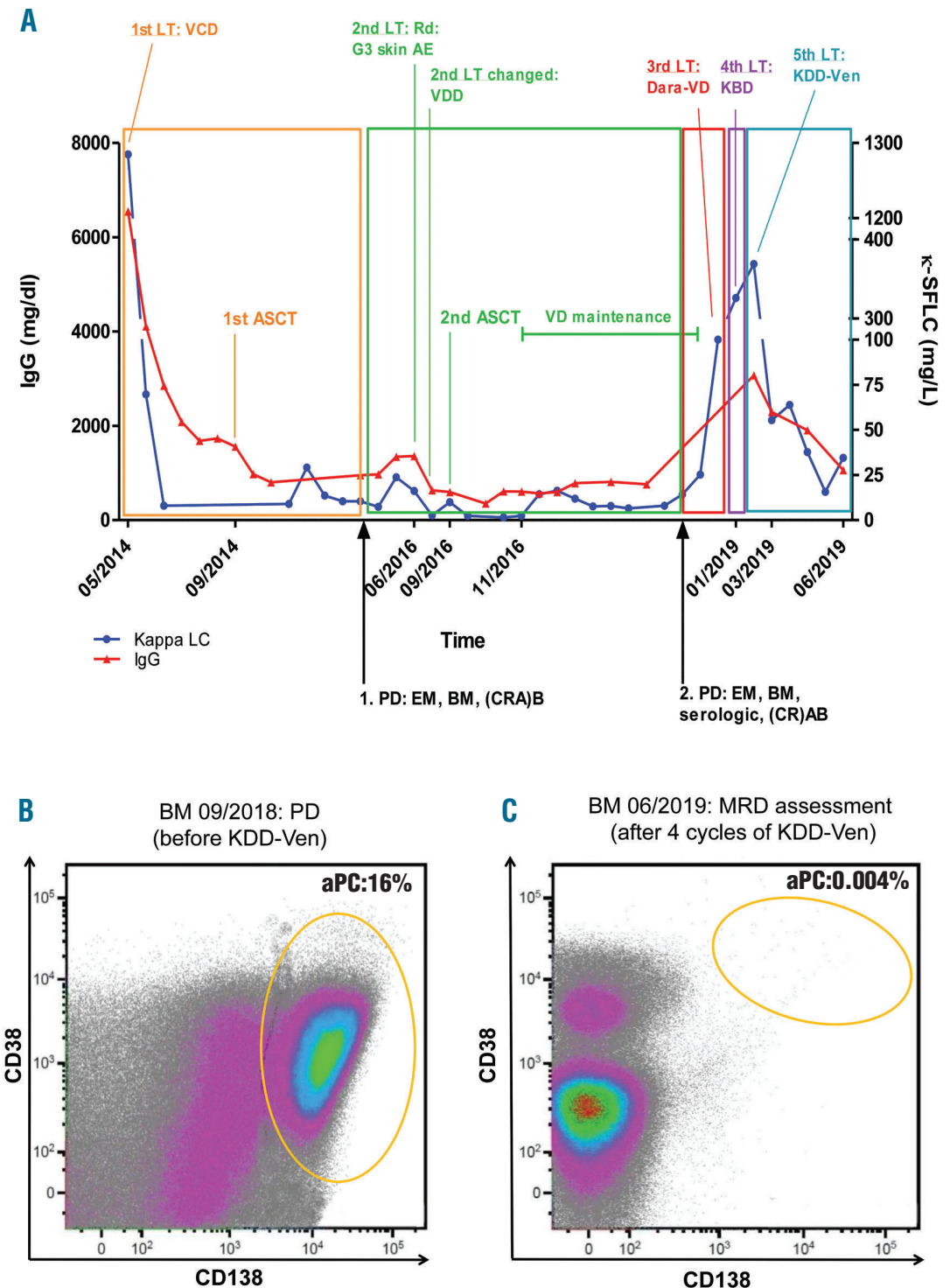

Figure 1. The clinical course of myeloma (MM) in this patient with notable findings. (A) Course of clinical parameters between $05 / 2014$ and $06 / 2019$ with performed first to fifth line antimyeloma therapy regimens. (B) Dot-plot of the flow cytometry analysis showing the CD138/CD38 gate in 09/2018, when disease progression reoccurred. Aberrant plasma cells (aPC) are depicted within the yellow-orange circle. (C) Dot-plot of the flow cytometry analysis showing the CD138/CD38 gate in 06/2019 with response to KDD-low-dose (100 mg/day) continuous venetoclax treatment. aPC are depicted within the yellow-orange circle. AE: adverse event; ASCT: autologous peripheral blood stem cell transplantation; B: bendamustin; BM: bone marrow; CRAB: hypercalcemia, renal failure, anemia, bone lesions; d: dexamethasone reduced; D: dexamethasone; D: doxorubicin; Dara: daratumumab; EM: extramedullary; IgG: immunoglobulin G; K: carfilzomib; K-SFLC: kappa serum free light chains; LT: line therapy, PD: progressive disease; R: lenalidomide, V: bortezomib; VCD: bortezomib, cyclophosphamide, dexamethasone; VD: bortezomib, dexamethasone; VDD: bortezomib, doxorubicin, dexamethasone; Ven: venetoclax. 
nation treatments have been described with striking results, especially in RRMM patients with $\mathrm{t}(11 ; 14)$.

Notable in our patient was the aggressive, unfavorable MM course with high BM infiltration, EM relapses, partial loss of paraprotein secretion with $\mathrm{MM}$ progression and extensive skin-SAE upon lenalidomide-treatment. ${ }^{8}$ Moreover, he showed refractoriness to V, KBD and Dara, and harbored two unfavorable cytogenetic features: one amplified IGH-CCND1 fusion ${ }^{18}$ and one gain 1q21, which is currently discussed as a potentially high risk feature for resistance through the upregulation of MCL-1. Nevertheless, combined treatment with KDD-low-dose venetoclax resulted in an impressive response (VGPR), which has been ongoing and is most probably related to venetoclax (Figure $1 \mathrm{~A}+\mathrm{C}$ ). In conclusion, this case suggests that this quadruplet was well-tolerated and that even low doses of venetoclax may induce deep response as shown via BM cytology, histology and MCF (Figure $1 C$ ) in otherwise difficult to treat RRMM patients, with substantial gain of their QoL and subsequent therapies being possible again, such as allogeneic transplants, ${ }^{19}$ bispecific $\mathrm{T}$-cell engager (BITE) or chimeric antigen receptor (CAR) T-cells. ${ }^{20}$

Sarolta Bojtine Kovacs, ${ }^{1 *}$ Jingting Luan, ${ }^{1 *}$ Sandra Maria Dold, ${ }^{1,2}$ Andreas Weis, ${ }^{1,3}$ Milena Pantic, Justus Duyster, ${ }^{1,3}$ Ralph Wäsch ${ }^{1,3}$ and Monika Engelhardt ${ }^{1,3 *}$

${ }^{*} S B K, J L$ and $M E$ contributed equally

'Departments of Medicine 1, Hematology and Oncology, Medical Center, Faculty of Medicine, University of Freiburg; ${ }^{2}$ Faculty of Biology, University of Freiburg; ${ }^{3}$ Comprehensive Cancer Center Freiburg (CCCF), Medical Center, Faculty of Medicine, University of Freiburg, Freiburg, Germany

Acknowledgments: the authors thank distinguished IMWG, EMN, $D S M M$ and GMMG myeloma experts for their advice, recommendations and insightful, inspiring comments.

Funding: this work was supported by the Deutsche Krebshilfe (grants 1095969 and 111424 [to ME and RW]).

Correspondence: MONIKA ENGELHARDT monika.engelhardt@uniklinik-freiburg.de doi:10.3324/haematol.2019.232330

Information on authorship, contributions, and financial \& other disclosures was provided by the authors and is available with the online version of this article at www. haematologica.org.

\section{References}

1. Moreau P, Touzeau C. Multiple Myeloma: From Front-Line to Relapsed Therapies. Am Soc Clin Oncol Educ Book. 2015:e504 e511.

2. Green DR, Walczak H. Apoptosis therapy: driving cancers down the road to ruin. Nat Med. 2013;19(2):131-133.

3. Bodet L, Ménoret E, Descamps G, et al. BH3-only protein Bik is involved in both apoptosis induction and sensitivity to oxidative stress in multiple myeloma. Br J Cancer. 2010;103(12):1808-1814.

4. Kumar S, Kaufman JL, Gasparetto C, et al. Efficacy of venetoclax as targeted therapy for relapsed/refractory $\mathrm{t}(11 ; 14)$ multiple myeloma. Blood. 2017;130(22):2401-2409.
5. Einsele H, Engelhardt M, Tapprich C, et al. Phase II study of bortezomib, cyclophosphamide and dexamethasone as induction therapy in multiple myeloma: DSMM XI trial. Br J Haematol. 2017;1 79(4):586-597.

6. Einsele $\mathrm{H}$, Knop S, Vogel M, et al. Response-adapted consolidation with bortezomib after ASCT improves progression-free survival in newly diagnosed multiple myeloma. Leukemia. 2017;31(6):14631466.

7. Straka C, Knop S, Vogel M, et al. Bortezomib consolidation following autologous transplant in younger and older patients with newly diagnosed multiple myeloma in two phase III trials. Eur J Haematol. 2019 June 24. [Epub ahead of print].

8. Wäsch R, Jakob T, Technau K, Finke J, Engelhardt M. StevensJohnson/toxic epidermal necrolysis overlap syndrome following lenalidomide treatment for multiple myeloma relapse after allogeneic transplantation. Ann Hematol. 2012;91(2):287-289.

9. Ludwig H, Adam Z, Hajek R, et al. Light Chain-induced acute renal failure can be reversed by bortezomib-doxorubicin-dexamethasone in multiple myeloma: results of a Phase II Study. J Clin Oncol. 2010; 28(30):4635-4641

10. Waldschmidt JM, Keller A, Ihorst G, et al. Safety and efficacy of vorinostat, bortezomib, doxorubicin and dexamethasone in a phase I/II study for relapsed or refractory multiple myeloma (VERUMM study: vorinostat in elderly, relapsed and unfit multiple myeloma). Haematologica. 2018;103(10):e473-e479.

11. Roussel M, Hebraud B, Hulin C, et al. The Impact of lenalidomide, bortezomib, and dexamethasone treatment on health-related quality of life in transplant-eligible patients with newly-diagnosed multiple myeloma: results from the IFM/DFCI 2009 Trial. Blood. 2018; 132(Suppl 1):716

12. Mateos M-V, Spencer A, Nooka AK, et al. Daratumumab-based regimens are highly effective and well tolerated in relapsed or refractory multiple myeloma regardless of patient age: subgroup analysis of the phase 3 CASTOR and POLLUX studies. Haematologica. 2019 June 20. [Epub ahead of print].

13. Gramatzki M, Guenther A, Offidani M, et al. Carfilzomib, bendamustine, and dexamethasone (KBd) in advanced multiple myeloma: The EMN09-trial. J Clin Oncol. 2018;36(15_suppl):8019.

14. Engelhardt M, Domm A-S, Dold SM, et al. A concise revised Myeloma Comorbidity Index as a valid prognostic instrument in a large cohort of 801 multiple myeloma patients. Haematologica. 2017;102(5):910-921.

15. Engelhardt M, Dold SM, Ihorst G, et al. Geriatric assessment in multiple myeloma patients: validation of the International Myeloma Working Group (IMWG) score and comparison with other common comorbidity scores. Haematologica. 2016;101(9):1110-1119.

16. Moreau P, Chanan-Khan A, Roberts AW, et al. Promising efficacy and acceptable safety of venetoclax plus bortezomib and dexamethasone in relapsed/refractory MM. Blood. 2017;130(22):2392-2400.

17. Kuhn DJ, Chen Q, Voorhees PM, et al. Potent activity of carfilzomib, a novel, irreversible inhibitor of the ubiquitin-proteasome pathway, against preclinical models of multiple myeloma. Blood. 2007;110(9):3281-3290

18. Ishigaki T, Sasaki K, Watanabe K, et al. Amplification of IGH/CCND1 fusion gene in a primary plasma cell leukemia case. Cancer Genet Cytogenet. 2010;201(1):62-65.

19. Greil C, Engelhardt M, Ihorst G, et al. Allogeneic transplantation of multiple myeloma patients may allow long-term survival in carefully selected patients with acceptable toxicity and preserved quality of life. Haematologica. 2019;104(2):370-379.

20. Raje N, Berdeja J, Lin Y, et al. Anti-BCMA CAR T-Cell Therapy bb2121 in relapsed or refractory multiple myeloma. N Engl J Med. 2019;380(18):1726-1737. 